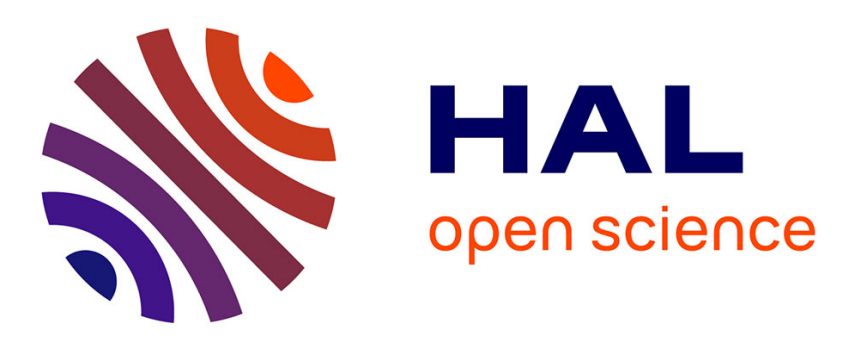

\title{
De ante à devant et avant : différenciation sémantique dans l'évolution des langues romanes
}

Benjamin Fagard

\section{To cite this version:}

Benjamin Fagard. De ante à devant et avant : différenciation sémantique dans l'évolution des langues romanes. Langages, 2012, 188 (4), pp.111-131. halshs-01241417

\section{HAL Id: halshs-01241417 https://shs.hal.science/halshs-01241417}

Submitted on 10 Dec 2015

HAL is a multi-disciplinary open access archive for the deposit and dissemination of scientific research documents, whether they are published or not. The documents may come from teaching and research institutions in France or abroad, or from public or private research centers.
L'archive ouverte pluridisciplinaire HAL, est destinée au dépôt et à la diffusion de documents scientifiques de niveau recherche, publiés ou non, émanant des établissements d'enseignement et de recherche français ou étrangers, des laboratoires publics ou privés. 
Benjamin Fagard

Lattice (CNRS, ENS \& Université Paris 3 Sorbonne Nouvelle)

De ante à devant et avant : différenciation

sémantique dans l'évolution des langues romanes

\section{INTRODUCTION}

Cet article porte sur l'évolution de la préposition latine ante. Nous partons de l'étude de cette préposition pour réfléchir à la problématique proposée comme point de départ de ce numéro : unicité et variabilité. À l'unicité du point de départ envisagé ante - s'opposent, d'une part, la polysémie de ce terme, qui a en latin classique des emplois spatiaux, temporels et abstraits (comme on peut s'y attendre d'après ce que l'on sait sur la grammaticalisation de prépositions spatiales ${ }^{1}$ signifiant "devant ", cf. Svorou 1994; Haspelmath 1997; Heine \& Kuteva, 2002 : 129, 141-142) ; d'autre part, la multiplicité et la variété de ses héritiers, comme nous allons le voir plus bas. Cette problématique rencontre ainsi celle du renouvellement des paradigmes prépositionnels entre latin et langues romanes, avec deux tendances contradictoires, spécialisation sémantique d'un côté, évolution vers une polysémie plus grande de l'autre. Nous proposons ici d'étudier le processus complexe d'évolution morphologique et sémantique dans le cas de ante, pour tenter de voir dans quelle mesure les phénomènes observables dans les différentes langues romanes sont comparables : les deux tendances sont-elles toujours présentes, ou bien peut-on observer des 'cycles', avec des phases de désambiguïsation ${ }^{2}$ et des phases d'évolution sémantique?

\footnotetext{
${ }^{1}$ La notion de préposition 'spatiale' a souvent été remise en cause (voir par exemple Cadiot 2002). Notre vision est proche de celle de Vandeloise (1985, 1986), qui montre bien que ces prépositions (devant, dans, sous, sur...) ne sont pas 'que' spatiales, et que l'on peut avec profit proposer pour les décrire des définitions plus fonctionnelles que géométriques. Par commodité, nous continuerons ici à parler de prépositions spatiales, au sens où elles sont bien utilisées entre autres pour décrire des situations spatiales. Cela ne signifie pas pour autant que nous leur attribuons un 'noyau sémantique' spatial.

${ }^{2}$ Ce type de phénomène a été décrit pour d’autres langues, ainsi du grec $\mu \varepsilon \tau \alpha$, polysémique ( «avec » et « après »), dont Luraghi (2005) montre qu'il s'est scindé en deux prépositions monosémiques, avec différenciation
} 
$\mathrm{Du}$ point de vue formel, les renforcements de ante apparaissent dans toutes les langues romanes : français devant, davant, avant; italien avanti, davanti, innanzi, dinanzi; espagnol enante, delante, adelante, antes; portugais antes, perante, diante; catalan et occitan abans, davant, enan(s); sarde anànti, daenànte, àntes ; roumain înainte(a); romanche aunz, avant, davant - sans compter les formes composées. On trouve, de plus, des grammaticalisations récentes qui remplissent des fonctions similaires dans plusieurs langues romanes, notamment pour les emplois spatiaux: français en face de, face à, vis-à-vis de; portugais em frente de ; espagnol frente $a$; italien di fronte $a$, dirimpetto $a$; roumain în faţă, etc. $^{33}$ La situation est donc complexe, notamment parce que les formes renforcées ne sont pas toujours équivalentes sémantiquement à ante; le plus souvent, même lorsqu'elles sont polysémiques, au moins un des domaines sémantiques (spatial, temporel, notionnel) est réservé à des usages littéraires ou vieillis. Les grammaticalisations plus récentes, elles, ne donnent pas lieu aux mêmes extensions sémantiques, et ne peuvent pas être employées dans le domaine temporel.

Après une brève introduction de la problématique du renouvellement prépositionnel (§ 2 ), nous décrivons la méthode adoptée pour la présente étude, à savoir les corpus retenus (§ 3) et l'analyse sémantique ( $\S 4$ ). Nous présentons ensuite les principaux résultats de l'étude ( $§ 5$ ), avant de conclure (§6).

\section{RENOUVELLEMENT DES PRÉPOSITIONS}

D'un point de vue morphosyntaxique, on sait que les langues romanes ont largement renouvelé leurs paradigmes prépositionnels, mais de manière en grande partie parallèle, avec le développement de nouvelles catégories de prépositions - fonctionnelles (à, de, en...), lexicales (avant, devant, vers, dans...), complexes (à l'égard de, face à, jusqu'à...) auxquelles sont associés divers degrés de fréquence et de grammaticalisation (Fagard \& Mardale 2007). D’un point de vue sémantique, il est plus difficile de généraliser, parce que l'on observe deux tendances contradictoires: d'un côté, le développement de nouveaux sens pour des formes anciennes,

formelle en $\mu \varepsilon$ (« avec ») et $\mu \varepsilon \tau \alpha$ (« après »).

${ }^{3}$ On pourrait poursuivre avec a cara $a$ en sarde (logudorese, cf. Corda, 1994 : 99) ou encore, pour les emplois temporels, l'italien prima di ; dresser une liste exhaustive de ces grammaticalisations n'est cependant pas l'objet de cette étude. Un aspect particulièrement intéressant de ces grammaticalisations, mais que nous ne pouvons malheureusement pas traiter ici, est leur caractère sinon universel du moins largement répandu; on retrouve, en effet, ce type de formations dans de nombreuses langues africaines (voir Batic 2010 ; Heine \& Reh 1982). 
avec une polysémie exponentielle, en particulier pour les prépositions les plus grammaticalisées (voir par exemple Kilroe 1994 pour la préposition à); de l'autre, l'apparition de nouvelles formes. Ces formes peuvent résulter de nouvelles grammaticalisations : français chez sur casa « maison », lés sur latus « flanc », vu sur le verbe voir ; occitan doumaci composé de dou « dieu » et maci sur mercede $(m)$ " salaire »; italien tranne sur trarre " tirer » et ne du latin inde " de là ", tramite sur tramite $(m)$ « chemin, sentier »... La grammaticalisation peut également concerner des adverbes, eux-mêmes formés par ajouts sur une base déjà grammaticale: post $\rightarrow$ postea, *postius $>$ puis ; ante $\rightarrow$ antea, *antius $>$ anzi. Les formes ainsi obtenues ne sont pas sémantiquement équivalentes aux formes de base, mais semblent avoir généralement un sémantisme plus réduit (ainsi de l'adverbe anteā, uniquement temporel, cf. Ernout \& Meillet 1967). Elles peuvent également résulter d'ajouts morphologiques sur une base déjà grammaticalisée, à l'aide d'une préposition 'préfixée', généralement en, de ou par : français envers, devers, pardevers; italien inverso ; espagnol debajo... Ces nouvelles formes entraînent souvent une spécialisation sémantique des formes existantes, avec la répartition des sens existants entre l'ancienne forme et la ou les nouvelle(s) (Fagard 2010). C'est ce dernier type de formation que nous étudions ici.

Concernant la préposition latine ante, la question que nous posons dans cet article est la suivante :

Quelles réélaborations morphologiques peut-on observer dans les langues romanes ? Dans quelle mesure les nouvelles formes obtenues participent-elles à la redistribution des sens de l'étymon, et donc à la réduction de la polysémie des formes préexistantes?

Il n'est pas si aisé qu’il y paraît de répondre à ces questions, parce que les formes sont variées (avec préfixation et suffixation récursives) et que, de plus, l'évolution sémantique se croise avec la différenciation syntaxique en préfixe, préposition et adverbe. Pour illustrer cette difficulté, nous indiquons plus bas les principales formes adverbiales et prépositionnelles issues du latin ante, dans les langues romanes médiévales (Tableau 1) et modernes (Tableau 2) ${ }^{4}$.

Tableau 1: Principales formes adverbiales et prépositionnelles issues de ante, dans les langues romanes médiévales

\footnotetext{
${ }^{4}$ Les corpus de langue médiévale contiennent des textes des XIIIe et XIVe siècles, sauf pour le français (du XIe au XVe); les corpus de langue moderne contiennent uniquement des textes du XXe siècle.
} 


\begin{tabular}{|c|c|c|c|c|c|}
\hline & $\begin{array}{l}\text { forme } \\
\text { de } \\
\text { base } \\
\end{array}$ & préfixation & suffixation & $\begin{array}{l}\text { affixation } \\
\text { multiple }\end{array}$ & $\begin{array}{l}\text { construction } \\
\text { complexe }\end{array}$ \\
\hline français & & avant & $\begin{array}{c}\text { ainz, } \\
\text { ainçois }\end{array}$ & $\begin{array}{c}\text { devant, } \\
\text { davant, } \\
\text { dedevant }\end{array}$ & $\begin{array}{c}\text { au devant } \\
\text { de, } \\
\text { paravant, } \\
\text { par devant, } \\
\text { auparavant }\end{array}$ \\
\hline italien & ante & avanti & anzi & $\begin{array}{c}\text { innanzi, } \\
\text { dinnanzi, } \\
\text { davanti }\end{array}$ & \\
\hline espagnol & ante & & antes & delante & $\begin{array}{c}\text { por delante, } \\
\text { pordelante } \\
\text { de }\end{array}$ \\
\hline portugais & ante & & antes & $\begin{array}{c}\text { perante, } \\
\text { adiante, } \\
\text { diante }\end{array}$ & diante de \\
\hline $\begin{array}{l}\text { occitan, } \\
\text { catalan }\end{array}$ & ant & enan, avan & $\begin{array}{l}\text { ans, } \\
\text { ancian }\end{array}$ & $\begin{array}{l}\text { davan(t), } \\
\text { abans, } \\
\text { anans, } \\
\text { enans }\end{array}$ & d'abans \\
\hline
\end{tabular}

Tableau 2: Principales formes adverbiales et prépositionnelles issues de ante, dans les langues romanes modernes

\begin{tabular}{|c|c|c|c|c|c|}
\hline & $\begin{array}{l}\text { forme } \\
\text { de } \\
\text { base }\end{array}$ & préfixation & suffixation & $\begin{array}{l}\text { affixation } \\
\text { multiple }\end{array}$ & $\begin{array}{l}\text { construction } \\
\text { complexe }\end{array}$ \\
\hline français & & avant & & devant & $\begin{array}{l}\text { par devant, } \\
\text { au-devant }\end{array}$ \\
\hline italien & ante & avanti & anzi & $\begin{array}{l}\text { davanti, } \\
\text { innanzi, } \\
\text { dinnanzi }\end{array}$ & \\
\hline espagnol & ante & & antes & $\begin{array}{l}\text { delante, } \\
\text { adelante }\end{array}$ & $\begin{array}{l}\text { por delante } \\
\text { de }\end{array}$ \\
\hline portugais & ante & & antes & $\begin{array}{l}\text { diante, } \\
\text { adiante, } \\
\text { perante }\end{array}$ & $\begin{array}{l}\text { por diante, } \\
\text { paradiante, } \\
\text { em diante }\end{array}$ \\
\hline occitan & & & abans & davant & \\
\hline catalan & & & abans & davant & \\
\hline roumain & & înainte & înaintea & & \\
\hline romanche & & avant & aunz & davant & \\
\hline sarde & & & $\begin{array}{l}\text { anànti, } \\
\text { àntes }\end{array}$ & daenànte & \\
\hline
\end{tabular}

Certaines de ces formes peuvent être employées comme adverbes ou conjonctions (cf. Raynouard, 1977: 87), et quelques-unes n'ont jamais ou presque d'emploi prépositionnel, ainsi de l'ancien français ainz. Nous nous intéresserons dans cet article principalement aux formes qui ont des emplois prépositionnels fréquents, sans faire la distinction entre emplois prépositionnels simples et constructions complexes où le complément de la préposition est introduit par de $(d i, d a)$, en 
(in, em) ou à (a), comme en (1) :

(1) Pasan las tropas peruanas por delante del palco oficial

" les trouves péruviennes passent devant la tribune officielle » (Corpus del Español, David Viñas, Los hombres de a caballo, XXe s.)

\section{MÉTHODOLOGIE : LES CORPUS}

Afin de retracer précisément l'évolution de ces formes prépositionnelles, nous partons des outils philologiques traditionnels ${ }^{5}$. Mais pour évaluer l'importance relative des différents sens, il nous faut observer les emplois en contexte, à l'aide d'études sur corpus. En effet, comme le souligne E. De Felice (1958 : 346), le contexte a une importance fondamentale dans l'évolution sémantique des prépositions ${ }^{6}$. Les périodes retenues pour cette étude sont les suivantes : ancien français, moyen français et français moderne; italien, espagnol et portugais anciens et modernes. Nous laissons de côté pour cette partie de l'étude les langues pour lesquelles nous ne disposons pas de corpus comparable : le roumain, le catalan, le romanche et le sarde. Pour chaque période, chaque langue et chaque forme (morphologique, non graphique) retenues, l'étude a été faite dans la mesure du possible sur une centaine d'occurrences extraites de corpus principalement littéraire ${ }^{7}$. Ceci nous permet de donner une image plausible de l'évolution sémantique des différentes formes, et de les comparer plus finement entre elles (voir notamment à ce propos Rinke \& Elsig 2010).

Nous présentons plus bas des données de fréquence pour l'ensemble des formes retenues, ainsi que le nombre d'emplois prépositionnels codés sémantiquement. Le nombre de ces occurrences est très limité pour certaines formes, qui sont

\footnotetext{
${ }^{5}$ Les principales sources utilisées sont les suivantes: Buridant (2000); Torres \& Carlos Assunção (2000) ; Bonomi (1986) ; Fernández González (1985) ; Liver (1982) ; Corominas (1980-1987) ; Marchello-Nizia (1974) ; Nunes (1969) ; Hescott (1961); Sävborg (1941) ; Ronjat (1980 [19301941]) ; Wartburg (1937-1970) ; Tobler \& Lommatzsch (1925) ; Raynouard (1977 [1838]).

6 Selon cet auteur, " l'acquisition d'une nouvelle valeur syntacticosémantique, autrement dit l'extension du noyau fondamental (de sens) d'une préposition, n'est semble-t-il quasiment jamais due à des forces endogènes, à une prolifération interne du noyau lui-même, mais à un glissement sémantique du verbe, de l'adjectif ou encore du substantif qui apparaît dans le syntagme, puis à l'enracinement et à la généralisation du nouveau type syntagmatique dans lequel cette valeur se trouve réalisée " (nous traduisons).

${ }^{7}$ Il serait intéressant de faire l'étude sur des corpus plus diversifiés, en incluant notamment des textes techniques, ou encore de l'oral pour les langues modernes; il nous semble cependant que la comparabilité ne peut être obtenue qu'à ce prix.
} 
surtout employées comme adverbes ou conjonctions. Pour des raisons de lisibilité, nous indiquons systématiquement la fréquence brute, mais aussi la fréquence relative, en nombre d'occurrences pour un million de mots. Cette donnée n'est pas nécessairement comparable pour les différentes langues, ni révélatrice de la fréquence « en langue », puisqu'il ne s’agit que de corpus; cependant, nous considérons qu'il s'agit d'une première indication utile.

Pour le portugais médiéval et moderne, nous avons utilisé le Corpus do português (désormais $\mathrm{CdP}$ ). Pour la période médiévale, plus précisément les XIIIe et XIVe siècles, les principales formes issues de ante sont les suivantes :

Tableau 3: Principales formes adverbiales et prépositionnelles issues de ante, en portugais médiéval

\begin{tabular}{|c|c|c|c|}
\hline $\begin{array}{c}\text { Forme } \\
\text { de base }\end{array}$ & $\begin{array}{c}\text { Nombre } \\
\text { d'occurrences }\end{array}$ & $\begin{array}{c}\text { Fréquence relative } \\
\text { (par million) }\end{array}$ & $\begin{array}{c}\text { Nombre d'occurrences } \\
\text { prépositionnelles analysées }\end{array}$ \\
\hline ante & 2290 & 1226,4 & 63 \\
\hline perante & 175 & 93,7 & 164 \\
\hline adiante & 57 & 30,5 & 75 \\
\hline diante (de) & 41 & 22,0 & $22^{\mathrm{b}}$ \\
\hline antes (de) & 21 & 11,2 & \\
\hline avante & 1 & 0,5 & \\
\hline
\end{tabular}

a. Calculées à partir de la taille du corpus pour les deux siècles (1 867236 mots, 550968 pour le XIIIe et 1316268 pour le XIVe s.).

b. Les nombres en italique indiquent que la fréquence n'est pas suffisamment élevée pour obtenir des résultats statistiquement exploitables. Nous laisserons donc de côté dans la suite de l'article les formes concernées.

Il faut ajouter à cela quelques occurrences avec des graphies divergentes, par exemple avec fusion de l'article (antel, perantel « devant lui »).

Pour le portugais moderne, les fréquences brutes sont sensiblement différentes, comme on le voit dans le Tableau 4 :

Tableau 4: Principales formes adverbiales et prépositionnelles issues de ante, en portugais moderne

\begin{tabular}{|c|c|c|c|}
\hline $\begin{array}{c}\text { Forme de } \\
\text { base }\end{array}$ & $\begin{array}{c}\text { Nombre } \\
\text { d'occurrences }\end{array}$ & $\begin{array}{c}\text { Fréquence } \\
\text { relative (par } \\
\text { million) }\end{array}$ & $\begin{array}{c}\text { Nombre d'occurrences } \\
\text { prépositionnelles analysées }\end{array}$ \\
\hline antes (de) & 10355 & 1034,6 & 158 \\
\hline diante (de) & 3605 & 360,2 & 152 \\
\hline perante & 1550 & 154,9 & 150 \\
\hline adiante & 788 & 78,7 & 147 \\
\hline ante & 539 & 53,9 & \\
\hline avante & 70 & 7,0 & \\
\hline
\end{tabular}


Nous analyserons les emplois des formes prépositionnelles, à savoir principalement diante, perante et ante. Antes, qui est principalement adverbial, a des emplois en tant que préposition composée qui sont presque exclusivement temporels. Adiante est presque exclusivement adverbial.

Pour le français médiéval (ancien et moyen français confondus), les fréquences des principales formes dans le corpus BFM sont présentées dans le Tableau 5 :

Tableau 5: Principales formes adverbiales et prépositionnelles issues de ante, en ancien et moyen français

\begin{tabular}{|c|c|c|c|}
\hline $\begin{array}{c}\text { Forme de } \\
\text { base }\end{array}$ & $\begin{array}{c}\text { Nombre } \\
\text { d'occurrences }\end{array}$ & $\begin{array}{c}\text { Fréquence relative } \\
\text { (par million) }\end{array}$ & $\begin{array}{c}\text { Nombre d'occurrences } \\
\text { prépositionnelles } \\
\text { analysées }\end{array}$ \\
\hline devant & 2440 & 791,5 & $1490(770+720)^{\mathrm{a}}$ \\
\hline avant & 1145 & 371,4 & $89(42+47)$ \\
\hline pardevant & 57 & 18,5 & $59(37+22)$ \\
\hline davant & 29 & 9,4 & $10(0+10)$ \\
\hline paravant & 16 & 5,2 & $4(0+4)$ \\
\hline audevant (de) & 11 & 3,6 & $31(1+30)^{\mathrm{b}}$ \\
\hline
\end{tabular}

a. Les chiffres donnés entre parenthèses correspondent respectivement à l'ancien et au moyen français.

b. Il faut en effet ajouter aux 11 occurrences de audevant une trentaine de séquences avec la graphie « au devant».

Nous analyserons dans la partie dédiée à la sémantique les formes avant, devant et pardevant.

Pour le français moderne, nous avons eu recours à la base de données FRANTEXT, qui permet de calculer l'évolution de la fréquence (absolue et relative) des différents mots étudiés. Il faut noter que la forme auparavant est exclusivement adverbiale.

Tableau 6 : Principales formes prépositionnelles issues de ante, en français moderne

\begin{tabular}{|c|c|c|c|}
\hline Forme de base & $\begin{array}{c}\text { Nombre } \\
\text { d'occurrences }\end{array}$ & $\begin{array}{c}\text { Fréquence relative } \\
\text { (par million) }\end{array}$ & $\begin{array}{c}\text { Nombre d'occurrences } \\
\text { prépositionnelles } \\
\text { analysées }\end{array}$ \\
\hline devant & 57862 & 542 & 148 \\
\hline avant & 60732 & 569 & 142 \\
\hline auparavant & 325 & 48 & \\
\hline pardevant & 0 & 0 & \\
\hline
\end{tabular}

Pour l'espagnol, nous avons utilisé le Corde et le Corpus del Español (désormais CdE). Pour la période médiévale, en 
particulier les XIIIe et XIVe siècles, les principales formes sont les suivantes (statistiques faites sur le CdE) :

Tableau 7 : Principales formes adverbiales et prépositionnelles issues de ante, en espagnol médiéval

\begin{tabular}{|c|c|c|c|}
\hline $\begin{array}{c}\text { Forme de } \\
\text { base }\end{array}$ & $\begin{array}{c}\text { Nombre } \\
\text { d'occurrences }\end{array}$ & $\begin{array}{c}\text { Fréquence relative } \\
\text { (taille du corpus : } \\
9746974 \text { mots) }\end{array}$ & $\begin{array}{c}\text { Nombre d'occurrences } \\
\text { prépositionnelles } \\
\text { analysées }\end{array}$ \\
\hline ante (de) & 13179 & 1352,1 & 118 \\
\hline antes $($ de) & 2435 & 249,8 & 100 \\
\hline delante $(a$, & 2263 & 232,2 & 73 \\
\hline
\end{tabular}

En espagnol moderne (Tableau 8), on retrouve les mêmes formes, avec des fréquences relatives assez différentes, puisque ante et delante sont nettement moins fréquents, tandis que antes l'est bien plus :

Tableau 8: Principales formes adverbiales et prépositionnelles issues de ante, en espagnol moderne

\begin{tabular}{|c|c|c|c|}
\hline $\begin{array}{c}\text { Forme de } \\
\text { base }\end{array}$ & $\begin{array}{c}\text { Nombre } \\
\text { d'occurrences }\end{array}$ & $\begin{array}{c}\text { Fréquence relative } \\
\text { (taille du corpus : } \\
20540 \text { 030 mots) }\end{array}$ & $\begin{array}{c}\text { Nombre d’occurrences } \\
\text { prépositionnelles } \\
\text { analysées }\end{array}$ \\
\hline antes (de) & 11643 & 566,8 & 190 \\
\hline ante & 7382 & 359,4 & 228 \\
\hline (por) delante & 1181 & 57,5 & 119 \\
\hline
\end{tabular}

Pour l'italien médiéval (Tableau 9), nous avons eu recours au corpus OVI. Les formes issues de ante présentent des fréquences relatives comparables à ce que nous avons trouvé pour les autres langues médiévales :

Tableau 9 : Principales formes adverbiales et prépositionnelles issues de ante, en italien médiéval

\begin{tabular}{|c|c|c|c|}
\hline Forme de base & $\begin{array}{c}\text { Nombre } \\
\text { d'occurrences }\end{array}$ & $\begin{array}{c}\text { Fréquence relative } \\
\text { (taille du corpus : } \\
22,3 \text { millions de } \\
\text { mots) }\end{array}$ & $\begin{array}{c}\text { Nombre d'occurrences } \\
\text { prépositionnelles } \\
\text { analysées }\end{array}$ \\
\hline $\begin{array}{c}\text { innanzi ( } a, d i, \\
\text { da) }\end{array}$ & 10845 & 486,3 & 380 \\
\hline $\begin{array}{c}\text { dinnanzi ( } a, d i, \\
\text { da) }\end{array}$ & 7140 & 320,2 & 452 \\
\hline anzi & 5590 & 250,7 & 33 \\
\hline $\begin{array}{c}\text { davanti (a,di, } \\
\text { da) }\end{array}$ & 2231 & 100,0 & 188 \\
\hline $\begin{array}{c}\text { avanti (a, di, } \\
\text { da) }\end{array}$ & 2140 & 96,0 & 3 \\
\hline ante & 98 & 4,4 & 23 \\
\hline
\end{tabular}

Pour l'italien moderne (Tableau 10), nous avons utilisé le corpus CoLFIS. Les fréquences sont dans l'ensemble plus 
faibles que pour le corpus médiéval :

Tableau 10 : Principales formes adverbiales et prépositionnelles issues de ante, en italien moderne

\begin{tabular}{|c|c|c|c|}
\hline Forme de base & $\begin{array}{c}\text { Nombre } \\
\text { d'occurrences }\end{array}$ & $\begin{array}{c}\text { Fréquence } \\
\text { relative }\end{array}$ & $\begin{array}{c}\text { Nombre d'occurrences } \\
\text { prépositionnelles }^{\text {analysées }}{ }^{\mathrm{a}}\end{array}$ \\
\hline davanti $(a, d a)$ & 1062 & 258,55 & 100 \\
\hline anzi & 635 & 144,95 & 0 \\
\hline avanti & 605 & 142,8 & 12 \\
\hline dinanzi $(a, d a)$ & 57 & 10,43 & 42 \\
\hline innanzi $(a)$ & 29 & 2,66 & 11 \\
\hline
\end{tabular}

a. En italien moderne standard, seule la forme davanti est à la fois fréquente et souvent prépositionnelle, ce qui explique le peu de données analysées pour les autres formes: avanti est rarement prépositionnelle, anzi jamais prépositionnelle, innanzi et dinanzi sont peu fréquentes.

\section{SÉMANTIQUE PRÉPOSITIONNELLE}

\subsection{Analyse sémantique}

L'analyse sémantique retenue pour cette étude est double: d'une part, pour le codage de chaque occurrence retenue, nous opérons une classification de base entre emploi en contexte spatial (2), emploi en contexte temporel (3) et emploi en contexte notionnel (4) :

(2) Le chien court / saute / est couché devant la voiture.

(3) Le facteur ne passe jamais avant $11 \mathrm{~h}$. / Il est arrivé avant toi.

(4) Il tremble devant l'ampleur de la tâche / Je suis consterné devant tant de bêtise.

On notera que ce qui est codé n'est pas uniquement le sens de la préposition elle-même, mais le sens du syntagme prépositionnel en contexte. L'analyse par type de contexte présente un intérêt certain pour une étude sémasiologique contrastive, dans la mesure où de nombreux contextes sont communs à plusieurs langues, comme en témoignent les exemples suivants :

- Établir un siège devant une ville

(5) Li rois d'Engleterre et la roine, quoique il fuissent a siege devant Calais, se apparilloient de grant poissance, et metoient ouvriers en oevre; et n'i avoit riens espargniet de cambres, d'abis, de rices jeuiauls, pour donner au jour des espousailles.

" Le roi d'Angleterre et la reine, bien qu'ils fussent au siège devant Calais... » (BFM, Froissart, Chroniques, XIVe s.)

(6) essendo l'assedio innanzi a Nequinia 
" tandis que le siège était mis devant Nequinia » (OVI, Boccace, Decameron, XIVe s.)

\section{- Se trouver devant la fontaine}

(7) A chelui point que li gaians tenoit en tel maniere le cevalier desous lui estoit mesire Tristrans devant la fontainne.

«... Tristan était devant la fontaine » (BFM, Tristan en prose, XIIIe siècle)

(8) [...] e davanti la fontana sì era lo primo petrone

" et il y avait ainsi devant la fontaine le premier gros rocher » (OVI, anonyme, Tavola ritonda, XIVe s.)

- Envoyer / plaider devant les juges

(9) Testamento de Costança Domigez, filha de Domjgue Anes, levado perante o juiz por Martim Balastro, procurador das donas de Chelas

« Testament... présenté devant le juge par Martin Balastro... » (CdP, Textos Notariais, XIVe s.)

(10) Il gentiluomo era più pregiato dalla madre, la quale desiderava di maritare molto altamente la sua figliuola: i tutori tenevano la parte dell'altro. E non potendosi accordare tra loro, elli furono dinanzi a' giudici. Quando i giudici intesero la richiesta della madre e de' tutori, elli giudicaro che le nozze si facessero a volontà della madre.

«... Et puisqu'ils n'arrivaient pas à se mettre d'accord, ils allèrent devant les juges. »

(OVI, anonyme, Deca prima di Tito Livio volgarizzata, milieu XIVe s.)

(11) La nonante et quatriesme nouvelle, d'ung curé qui portoit courte robe comme font ces galans a marier ; laquelle cause il fut cité devant son juge ordinaire, et de la sentence qui en fut donnée; aussi la deffense qui lui fut faicte, et des autres tromperies qu'il fist après, comme vous orrez plus a plain.

« un curé qui... fut cité devant son juge » (BFM, Cent nouvelles

nouvelles, milieu XVe s.)

- Être devant un problème

(12) Le ruego que, ante el menor problema, se ponga en contacto conmigo

« Je vous demande de me contacter pour tout problème, même mineur (litt. devant le plus petit problème) »

(CdE, Vargas Llosa, La Fiesta del Chivo, XXe s.)

(13) Je me retrouvais devant le même problème que tout à l'heure, sauf que cette fois-ci, la chance m'a souri, j'ai pas été obligé d'acheter qui que ce soit pour m'en sortir.

(Frantext, Djian, Le matin, XXe s.)

Le sens de la préposition n’est pas nécessairement toujours le 
même dans un contexte donné; ou plutôt, de subtils changements dans le contexte peuvent être associés à un changement du sens de la préposition, notamment par inférence (cf. Traugott \& Dasher 2002); on pourra ainsi se poser la question du sens précis de ante et avanti dans les deux exemples suivants, le premier étant plutôt spatial et le second plutôt fonctionnel (au sens de Vandeloise 1985, 1986) :

(14) el buen serujente que leuará tal presente ante Dios.

" le bon serviteur, qui amènera ce présent devant dieu "

(Corde, Veragüe, Pedro de Doctrina de la discripción o Tractado de la doctrina, milieu XIVe s.)

(15) tu avochata nostra avanti Dio

" toi, notre avocate auprès de dieu (litt. devant dieu) » (OVI, Enselmino da Montebelluna, XIVe s.)

Le second temps de l'analyse est un classement par domaine sémantique. Les emplois retenus pour cette étude sont liés à trois domaines sémantiques: emplois spatiaux, emplois temporels et emplois notionnels (où la préposition introduit généralement une relation de causalité) - voir les exemples (2), (3) et (4), respectivement. La définition que nous adoptons pour les emplois spatiaux inclut les emplois spatiaux fonctionnels, comme en (15), ou encore en (16-17) où le sens n'est pas purement géométrique :

(16) deve-se ter sempre diante dos olhos a imperfeição da criatura " on doit toujours avoir devant les yeux l'imperfection de la créature »(CdP, São Boaventura, XXe s.)

(17) A história dos vitoriosos devia mostrar a outra face da moeda e assim teríamos diante dos olhos a verdade mantida sem mentiras que obscurecem os padrões civilizatórios.

«... on aurait ainsi devant les yeux la vérité maintenue sans mensonges... » (CdP, Opinião do dia, XXe s.)

Elle inclut également certains emplois qui pourraient sembler métaphoriques, comme en (18), mais ne le sont pas de notre point de vue, parce qu'il s'agit d'une comparaison explicite (como perante : «comme devant... ») :

(18) - logo, encolhendo os ombros, como perante um obstáculo inevitável : - Mas que fazer...

" ensuite, haussant les épaules comme devant un obstacle inévitable, il soupira : '- Mais que faire...' "

(CdP, Manuel de Fonseca, Cerromaior, XXe s.)

Nous considérons, en revanche, que les emplois temporels lexicalisés (i.e. notés par les dictionnaires) ne doivent plus être compris comme métaphoriques. 
Il reste, comme toujours dans ce type d'analyse, des occurrences délicates à traiter. Pour systématiser l'étude et clarifier dès le départ les choix faits lors du codage, la méthode choisie est la suivante : lorsque le sens spatial est encore une des interprétations envisageables, l'occurrence est codée comme spatiale même s'il y a bien une inférence notionnelle (ici de type causal), comme dans les exemples (19) et (20) :

(19) Os dois homens riram, diante do espetáculo daquela mulher muito gorda e roliça, de pernas curtas

" les deux hommes se mirent à rire devant le spectacle de cette femme très grosse et potelée, aux jambes courtes "

(CdP, Cornélio Penna, A Menina Morta, XXe s.)

(20) - Par saint Pierre, dist li evesques, je wel bien que tuit cil de saienz sachent que je n'ais seignor ou monde fors l'apostoile en cui protencion je suy, ne devant autre seignor je ne respondroie.

"Par saint Pierre, dit l'évêque, je veux que tous ceux qui sont ici sachent que je n'ai pas de seigneur sauf l'apôtre qui me protège, et que je ne répondrai devant aucun autre seigneur »

(BFM, Ménestrel de reims, milieu XIIIe s.)

En revanche, certaines occurrences sont clairement non spatiales :

(21) Será essa a tão falada "fracassomania », sempre mencionada pelo presidente FHC ? Diante da perspectiva sombria, vou desistir de prestar o concurso vestibular de engenharia.

« Devant cette sombre perspective, je vais renoncer à me présenter au concours d'entrée d'ingénierie »

(CdP, Difusor cultural, XXe s.)

Parmi ces occurrences notionnelles, on trouve quelques occurrences isolées où la relation n'est pas exactement causale, comme dans l'exemple (22) :

(22) Qual è il valore di ogni singolo individuo davanti alle decisioni delle istituzioni ufficiali come i governi, i tribunali, le autorità che determinano il corso della Storia ? La risposta sta dentro di noi e dipende dalla coscienza individuale.

"Quelle est la valeur de chaque individu face aux décisions des institutions officielles...? »

(CoLFIS, Grazia, XXe s.)

\subsection{La question de la polysémie}

La catégorisation proposée concernant la polysémie permet d'en distinguer trois degrés possibles, faible (i), légère (ii) ou moyenne (iii) :

(i) emploi monosémique ou quasi monosémique, lorsque 
l'emploi le plus fréquent compte pour plus de $90 \%$ des emplois ;

(ii) emploi plutôt polysémique lorsque le ou les contexte(s) minoritaire(s) compte(nt) pour au moins $25 \%$ des emplois ;

(iii) emploi clairement polysémique si ces derniers comptent pour plus de $40 \%$ des emplois.

D'un point de vue théorique, une des questions que l'on peut se poser est celle du statut à accorder aux variations sémantiques que nous avons observées dans les corpus. Comme le notent G. Kleiber $(1999,2008)$ ou encore A. Blank (1999), il est difficile de faire la distinction entre variation contextuelle et polysémie (conçue comme une chaîne ou un réseau de sens, op. cit. : 1415). Cette distinction est pourtant fondamentale; pour y parvenir, ce dernier définit la variation contextuelle (qu'il appelle aussi vagueness) comme " l'actualisation, dans une occurrence donnée, du sens lexical du mot, malgré l'absence de certains traits définitoires » (op. cit. : 16). L'auteur illustre son propos par l'exemple de arm " bras » en anglais, qui présente à la fois des variations contextuelles (cf. 23-26) et une certaine polysémie (cf. 27-29) :

(23) my arm hurts

(24) look at the arm of the statue

(25) my mother was overdosed on LSD, so my arm is this little thing on my stomach

(26) a robotic arm reached out and grabbed me

(27) the arm of a coat

(28) the arm of a record player

(29) an arm of the sea

Appliquer cette définition de la variation contextuelle aux prépositions peut sembler complexe, car les traits sémantiques définitoires sont bien plus généraux et se prêtent davantage, semble-t-il, à la contextualisation. Il nous semble cependant qu'un élément fondamental est la question des transferts d'un domaine sémantique à l'autre : ces glissements sémantiques du spatial au temporel (devant les yeux > avant la fête), du spatial au notionnel (devant les yeux > devant la situation), sont généralement considérés comme des cas de métaphore, aboutissant à la création d'unités polysémiques. Lors de l'apparition d'un nouveau sens, si l'on se place dans le cadre théorique proposé par E. Traugott et R. Dasher (2002), on peut considérer qu'ils constituent des cas d'inférence invitée, plutôt que de variation contextuelle. Ils doivent alors être interprétés non plus comme des métaphores, mais comme des cas de 
métonymie - la métonymie étant comprise ici comme une opération conceptuelle (Hopper \& Traugott, 1993: 80-81). Ainsi, il n'y a pas de 'saut conceptuel' d'un domaine à l'autre, mais des glissements de sens dont on peut considérer, à l'instar de P. Koch (2001, 2004), qu'ils se font à l'intérieur d'un cadre donné. Cependant, une fois que le nouveau sens est lexicalisé, il y a bien polysémie, quel qu'en soit le mode d'apparition.

\section{PRINCIPAUX RÉSULTATS}

\subsection{Polysémie}

Nous commençons par une approche synchronique de la polysémie, en prenant chaque sous-corpus comme indicatif d'un état de langue ${ }^{8}$ : de ce point de vue, on peut établir une typologie des relations entre forme et sens observées pour les expressions issues du latin ante. Cela fait apparaître six types de formes: (a-b) clairement spatiales ou temporelles; (c-e) plutôt spatiales, temporelles ou notionnelles ; (f) peu marquées. Noter que les formes précédées d'une astérisque sont peu fréquentes: pour ces formes, les résultats sont donc donnés sous réserve.

- Formes (quasi-)monosémiques (95 \% ou plus pour un sens donné) :

(a) spatiales : perante (port. méd.), davanti (it. méd.), delante (esp. méd.), devant (moy. fr.), diante (port. méd.), *pardevant (moy. fr.)

(b) temporelles : avant (fr. mod.), antes (esp. méd., esp. mod., port. mod.), *antes (port. méd.), *anzi (it. méd.), *avanti (it. mod. $)^{9}$

- Formes peu polysémiques (75 \% ou plus pour un sens donné) :

(c) spatiales : davanti (it. mod.), dinnanzi (it. méd.), delante (esp. mod.), devant (anc. fr.), devant (fr. mod.), *davant (moy. fr.), *avanti (it. méd.)

(d) temporelle : avant (moy. fr.)

\footnotetext{
${ }^{8}$ Avec toutes les précautions que cela suppose : nous ne considérons pas les corpus comme des témoins fidèles d'un état de langue, mais comme indicateurs d'une tradition discursive qui est plus ou moins éloignée, selon les époques, de l'état de langue 'standard'. Cela est d'autant plus vrai qu'il s'agit de corpus écrits, par nature davantage marqués par la norme, et qu'il nous semble difficile de proposer des corpus de référence pour la langue médiévale.

${ }^{9}$ Mais il s'agit surtout d'emplois figés du type avanti Cristo « avant Jésus

Christ », dans les dates.
} 
(e) notionnelle : perante (port. mod.)

- Formes polysémiques (moins de $75 \%$ pour le sens le plus fréquent) :

(f) spatio-notionnelles : diante (port. mod.), ante (port. mod.)

(g) spatio-temporelles : innanzi (it. méd.), avant (anc. fr.), *paravant (moy. fr.), *ante (it. méd.)

On constate donc une prévalence assez large des formes (a-e), peu polysémiques ou quasi-monosémiques, avec 15 formes (20 en comptant celles dont les emplois prépositionnelles sont très rares) contre 4 seulement (6 en comptant les formes rarement prépositionnelles) qui sont assez polysémiques (f). La comparaison entre période médiévale et période moderne semble indiquer que la tendance à la désambiguïsation (comme on peut l'observer pour vers, envers et devers en français, occitan et italien, cf. Fagard 2010) n'est pas spécialement à l'œuvre dans l'évolution sémantique de ces formes : en effet, la proportion de formes polysémiques augmente très nettement dans le temps (Tableau 11) :

Tableau 11 : Degré de polysémie des formes prépositionnelles issues de ante dans les corpus utilisés ${ }^{\mathrm{a}}$

\begin{tabular}{|c|c|c|}
\hline Degré de polysémie & Période médiévale & Période moderne \\
\hline $\begin{array}{c}\text { Faible } \\
\text { (de } 0 \text { à } 5 \% \text { pour les } \\
\text { sens minoritaires) }\end{array}$ & $\begin{array}{c}\text { antes (port., } 22 \text { осс.) } \\
\text { anzi (it., } 33 \text { осс.) } \\
\text { davanti (it., } 184 \text { осс.) } \\
\text { delante (esp., } 67 \text { осc.) } \\
\text { devant (moy. fr., } 762 \text { осc.) } \\
\text { diante (port., } 75 \text { осc.) } \\
\text { pardevant (moy. fr., } 5 \text { осс.) } \\
\text { perante (port., } 163 \text { осс.) } \\
\text { antes (esp., } 100 \text { осс.) }\end{array}$ & $\begin{array}{c}\text { antes (esp., } 190 \text { осс.) } \\
\text { antes (port., } 158 \text { осc.) } \\
\text { avant (fr., } 142 \text { occ.) }\end{array}$ \\
\hline $\begin{array}{c}\text { Léger } \\
\text { (de } 5 \text { à } 25 \% \text { pour les } \\
\text { sens minoritaires) }\end{array}$ & $\begin{array}{l}\text { avant (moy. fr., } 49 \text { осc.) } \\
\text { avanti (it., } 21 \text { occ.) } \\
\text { davant (moy. fr., } 10 \text { occ.) } \\
\text { devant (anc. fr., } 811 \text { occ.) } \\
\text { dinnanzi (it., } 424 \text { occ.) }\end{array}$ & $\begin{array}{c}\text { avanti (it., } 12 \text { осс.) } \\
\text { davanti (it., } 100 \text { осс.) } \\
\text { delante (esp., } 104 \text { осс.) } \\
\text { devant (fr., } 148 \text { осc.) } \\
\text { perante (port., } 149 \text { осс.) }\end{array}$ \\
\hline $\begin{array}{c}\text { Moyen } \\
\text { (plus de 25\% pour les } \\
\text { sens minoritaires) }\end{array}$ & $\begin{array}{c}\text { ante (esp., } 96 \text { occ.) } \\
\text { ante (it., } 3 \text { осc.) } \\
\text { ante (port., } 63 \text { occ.) } \\
\text { avant (anc. fr., } 44 \text { occ.) } \\
\text { innanzi (it., } 87 \text { occ.) } \\
\text { paravant (moy. fr., } 2 \text { occ.) }\end{array}$ & $\begin{array}{c}\text { ante (esp., } 228 \text { осс.) } \\
\text { ante (port., } 146 \text { осс.) } \\
\text { diante (port., } 151 \text { осс.) } \\
\text { dinanzi (it., } 42 \text { осс.) }\end{array}$ \\
\hline
\end{tabular}

a. Certaines de ces formes n’introduisent pas leur complément directement, mais sont suivies d'une préposition fonctionnelles, parfois même de manière obligatoire, comme pour antes de. Pour ne pas surcharger le tableau, nous indiquons uniquement la forme de base.

Il semble en conséquence nécessaire de prendre en compte d'autres phénomènes pour décrire l'évolution sémantique des 
formes étudiées. C'est ce que nous tentons de faire dans la section suivante.

\section{2. Évolution sémantique et formelle}

On peut constater deux tendances principales dans l'évolution formelle et sémantique entre la période médiévale et la période moderne : d'une part, la perte des emplois prépositionnels pour certaines formes ; d'autre part, un glissement du spatial vers le notionnel.

Ainsi, entre l'espagnol médiéval et moderne, delante semble avoir conservé des emplois similaires, tandis que ante a perdu ses emplois temporels au profit d'emplois notionnels (Graphique 1). En espagnol comme en portugais, la préposition complexe antes de (dont les résultats ne sont pas présentés dans les graphiques) a dès l'époque médiévale des emplois exclusivement temporels, qu'elle conserve à l'époque moderne. Entre le portugais médiéval et moderne, ante, diante et perante ont glissé du spatial au notionnel (Graphique 2) ${ }^{10}$. On peut faire la même remarque concernant les prépositions de l'italien médiéval, à ceci près que plusieurs ont perdu leurs emplois prépositionnels, complètement (anzi) ou presque (avanti, innanzi) : les seules ayant conservé des emplois prépositionnels relativement fréquents ont bien connu un glissement du spatial vers le notionnel (Graphique 3). Ce glissement est perceptible également pour devant entre français médiéval et moderne, avec la perte des emplois temporels au passage, tandis que la préposition avant a perdu ses emplois spatiaux pour des emplois temporels (Graphique 4).

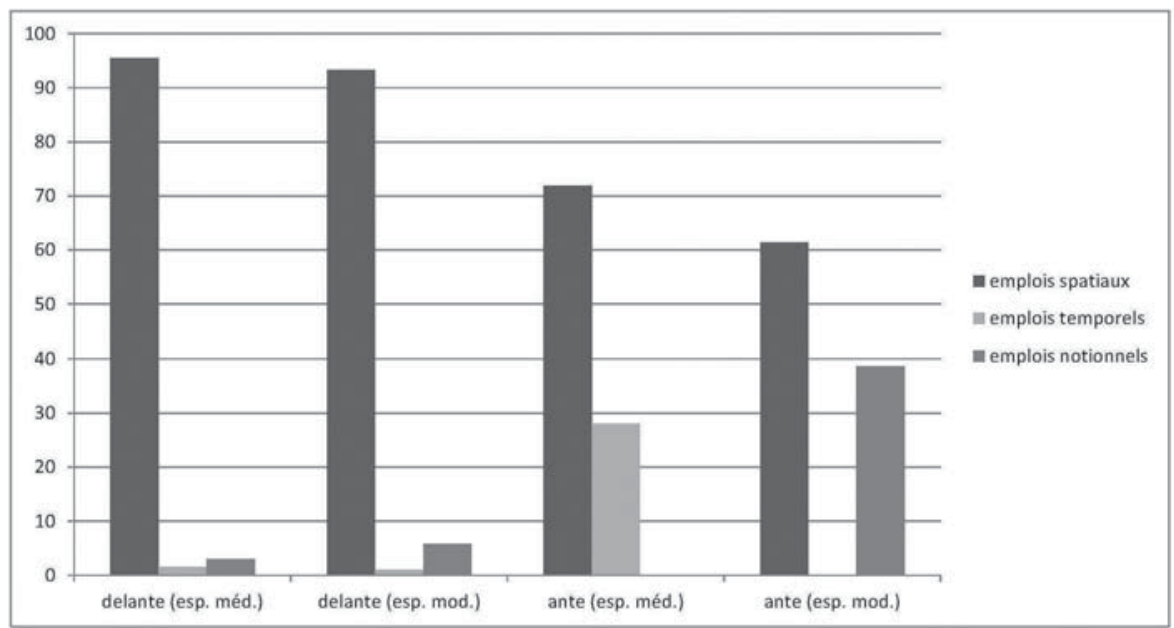

${ }^{10}$ Pour le portugais et l'espagnol, nous avons pu confirmer ces résultats à l'aide des outils proposés par les bases de données lemmatisées et codées morphologiquement Corpus del español et Corpus do português, qui permettent d'étudier assez finement les contextes d'apparition de différents mots (notamment à l'aide de la fonction « compare »). 
Figure 1 : Emplois prépositionnels des formes issues de ante en espagnol médiéval et moderne

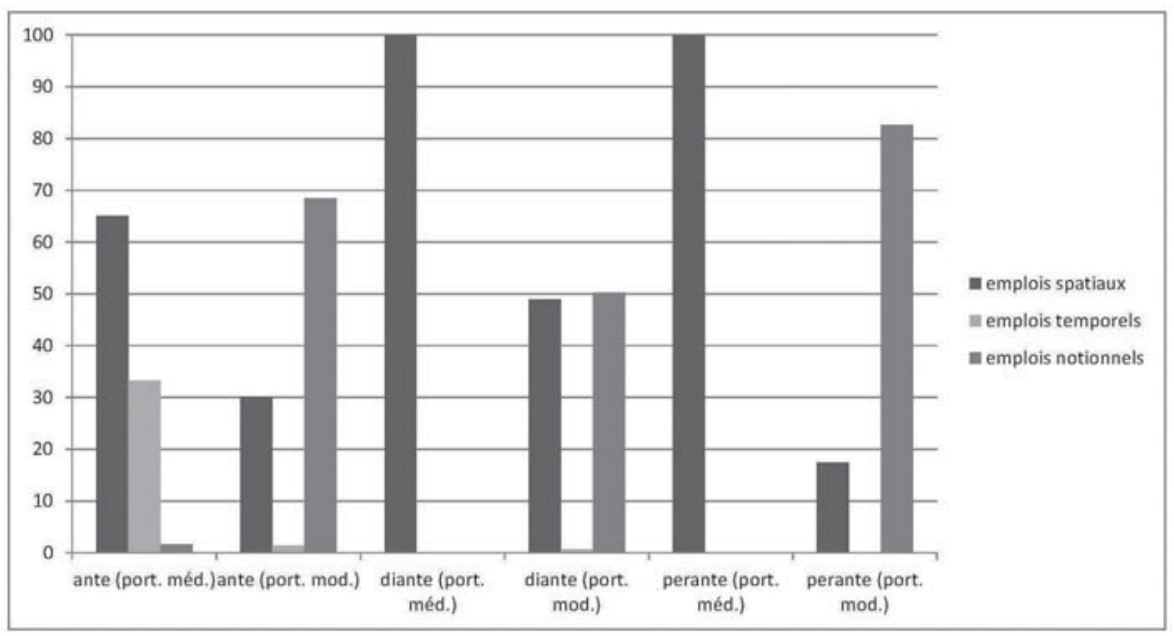

Figure 2 : Emplois prépositionnels des formes issues de ante en portugais médiéval et moderne

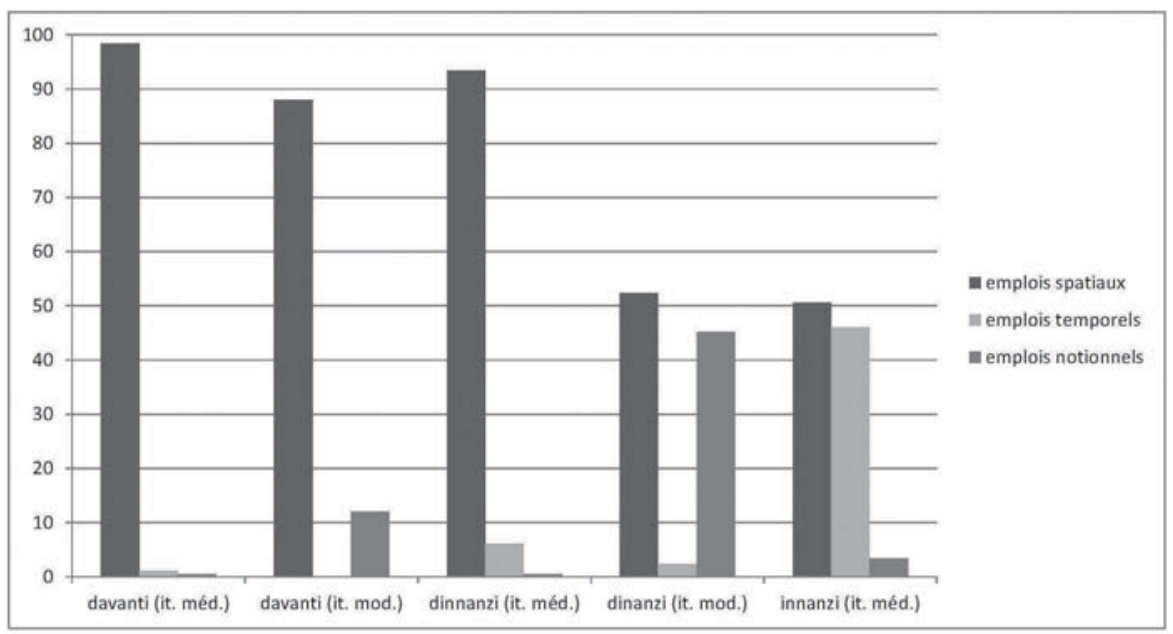

Figure 3: Emplois prépositionnels des formes issues de ante en italien médiéval et moderne

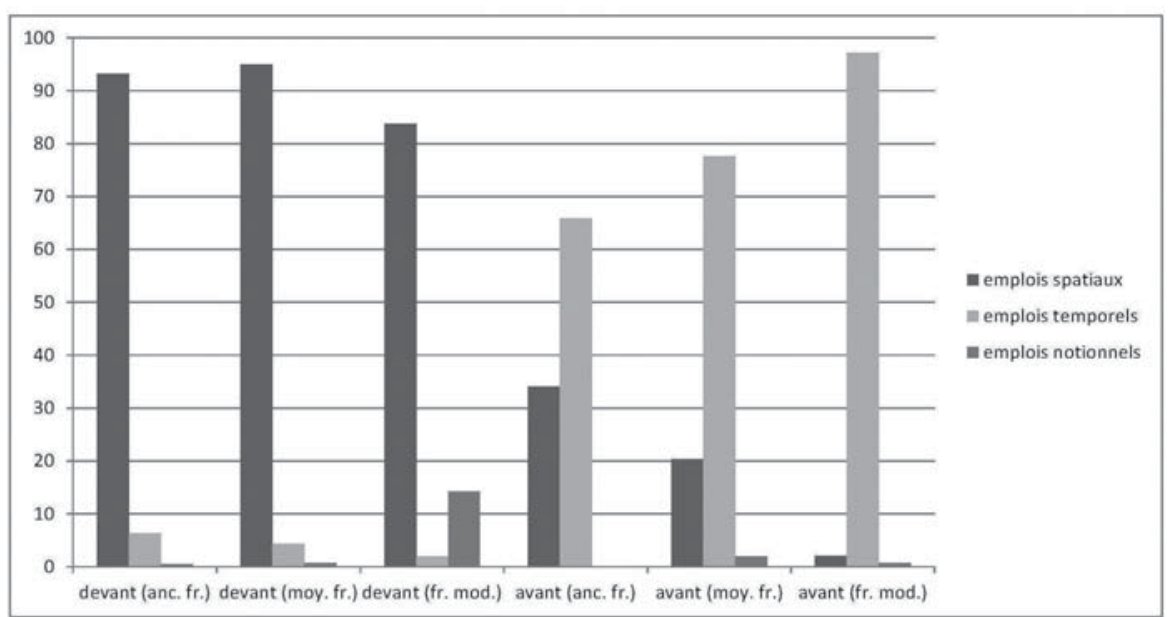


Figure 4: Emplois prépositionnels des formes issues de ante en français médiéval et moderne

\section{CONCLUSION}

L'évolution des formes prépositionnelles issues du latin ante dans les langues romanes est complexe et liée à un ensemble de phénomènes morphologiques, syntaxiques et sémantiques, quelle que soit la langue considérée. Nous avons ainsi pu observer un renouvellement formel par préfixation et suffixation, renforcé par l'apparition de formes complexes (avec la présence de prépositions fonctionnelles avant et/ou après la forme issue de ante), dans toutes les langues romanes : ces formes peuvent avoir des emplois comme adverbes, prépositions ou conjonctions, et tandis que certaines se spécialisent, d'autres conservent une assez grande liberté morpho-syntaxique. Pour quatre langues romanes, nous avons comparé les emplois prépositionnels dans la langue médiévale et la langue moderne, afin de mesurer l'importance relative de deux phénomènes qui nous semblent fondamentaux : l'évolution sémantique, qui peut produire des lexèmes polysémiques, et la spécialisation, répondant au principe d'isomorphie (cf. Hock \& Joseph, 1996 : 225). Ce principe s'est appliqué uniquement pour la séparation des formes temporelles, dès la période médiévale pour espagnol et portugais (dans ces deux langues, antes de apparaît d'emblée dans nos corpus avec des emplois exclusivement temporels), un peu plus tard pour le français et l'italien, devant et innanzi perdant leurs emplois temporels au profit de avant et (entre autres) prima di. Pour les formes spatiales et notionnelles, en revanche, le principe d'isomorphie n'a pas eu d'effet observable, le nombre de formes polysémiques ayant augmenté entre la période médiévale et la période moderne.

Ce qui semble avoir eu lieu, plutôt qu'une désambiguïsation, c'est un glissement sémantique global de toutes les formes ayant conservé des emplois prépositionnels. En effet, si l'on regarde de plus près les seules formes à la fois réellement polysémiques et dont les emplois prépositionnels sont relativement fréquents, nous avons deux prépositions principalement spatiales et temporelles (innanzi en italien médiéval et avant en ancien français), et deux autres principalement spatiales et notionnelles (ante et diante en portugais moderne). D'un point de vue diachronique, c'est assez surprenant : tandis que la seule forme ambiguë a disparu du français, deux formes sont devenues ambiguës en portugais moderne, et la dernière est restée ambiguë, de l’italien médiéval à l'italien moderne. Cependant, si l'on observe de plus près 
l'évolution de ces formes, on peut ramener ces différences à un même phénomène de perte des emplois spatiaux. Ainsi, avant n'est plus polysémique car il a perdu ses emplois spatiaux. De la même manière, on peut observer un glissement du spatial au notionnel pour ante: l'ambiguïté semble donc être un état transitoire entre une monosémie spatiale et une monosémie notionnelle, i.e. le passage d'une phase où le seul sens possible est spatial, à une autre où le seul sens possible est notionnel. Pour diante, la tendance est la même, puisque l'on ne trouve que des emplois spatiaux aux XIIIe et XIVe siècles dans notre corpus, tandis que l'on constate un net glissement vers les emplois notionnels en portugais moderne. Le cas d'innanzi est légèrement plus complexe: les emplois médiévaux sont spatiaux et temporels, mais les emplois modernes sont spatiaux et notionnels. Étant donné le faible nombre d'occurrences pour l'italien moderne (11 occurrences prépositionnelles seulement), il nous semble hasardeux de formuler des hypothèses sur l'évolution de cette forme, à ce stade de nos recherches. On notera que tous ces glissements du spatial au temporel et/ou au notionnel sont plus marqués encore dans les emplois non prépositionnels, en particulier dans les conjonctions (avant que, avant de, antes que, antes de, etc.), qui n'ont jamais d'emplois spatiaux $^{11}$.

La principale question qui se pose maintenant, selon nous, est de savoir dans quelle mesure les nouvelles formes - issues non de ante, mais de chaînes de grammaticalisation parallèles, avec des prépositions issues de noms désignant le front, le visage, etc. - entrent dans le même paradigme, avec des emplois parallèles aux formes issues de ante. En d'autres termes, les prépositions 'romanes' (espagnol frente a, français face à, etc.) utilisées désormais en concurrence avec les prépositions issues de ante pour les emplois " spatiaux » ontelles toutes la même tendance à acquérir des emplois non spatiaux ? C'est là une problématique qu'il nous reste encore à aborder.

\section{Références}

BATIC G. C. (2010), "Lexicon and Grammar of Space in West Chadic”, in G. Marotta et al. (eds), Space in Language, Pisa: Editrice Testi Scientifici, 433-445.

BLANK A. (1999), "Polysemy in the Lexicon", in R. Eckhardt \& K. von Heusinger (eds), Meaning Change - Meaning

\footnotetext{
${ }^{11}$ Cela ne vaut pas, cependant, pour les emplois nominaux, qui sont d'après nous à classer à part (l'avant du navire vs. *l'avant du spectacle). C'est plus ou moins vrai pour les emplois comme préfixe : avant-spectacle (temporel), avant-garde (sens à l'origine purement spatial, désormais plus complexe). Nous tenons à remercier les relecteurs anonymes et Dejan Stosic pour ces précisions.
} 
Variation, Workshop held at Konstanz, Vol. I, 11-29.

BONOMI I. (1986), Regole della lingua fiorentina (Giambullari, Pierfrancesco, 1546), Florence : Accademia.

BURIDANT C. (2000), Grammaire nouvelle de l'ancien français, Paris : Sedes.

CADIOT P. (2002), " Schémas et motifs en sémantique prépositionnelle: vers une description renouvelée des prépositions dites 'spatiales' ", Travaux de linguistique 44 (1), 9-24.

CORDA F. (1994), Grammatica moderna del sardo logudorese, Cagliari : Della Torre.

COROMINAS J. (1980-1987), Diccionario crítico etimológico castellano, Madrid : Editorial Gredos.

DE FELICE E. (1958), « La preposizione italiana $a$ », Studi di filologia italiana XVI, 343-409.

ERNOUT A. \& MEILLET A. (1967 [1932]), Dictionnaire étymologique de la langue latine - histoire des mots, Paris : Klincksiek (4e éd.).

FAGARD B. (2010), Espace et grammaticalisation L'évolution sémantique des prépositions dans les langues romanes, Sarrebruck : Éditions Universitaires Européennes.

FAGARD B. \& MARDALE A. (2007), « Systèmes prépositionnels des langues romanes : la notion de partie du discours en diachronie ", in D. Trotter (éd.), Actes du XXIVe Congrès International de Linguistique et Philologie Romanes, Vol. 1, Tübingen : Niemeyer, 91-105.

FERNÁNDEZ GONZÁLEZ J. R. (1985), Gramática Histórica Provenzal, Oviedo : Universidad de Oviedo.

HASPELMATH M. (1997), From space to time -Temporal adverbials in the World's Languages, München/Newcastle: Lincom Europa.

HEINE B. \& KUTEVA T. (2002), World lexicon of grammaticalization, Cambridge: Cambridge University Press.

HEINE B. \& REH M. (1982), Patterns of Grammaticalization in African Languages [Arbeiten des Kolner UniversalienProjektes 47], Cologne: Universität zu Köln, Institut für Sprachwissenschaft.

HESCOTT R. (1961), El desarrollo de las preposiciones latinas en español medieval, Mexico : Universidad nacional autónoma de México.

HOCK H. \& JOSEPH B. (1996), Language History, Language Change, Language Relationships, Berlin: Mouton de Gruyter.

HOPPER P. \& TRAUGOTT E. (1993), Grammaticalization, Cambridge: Cambridge University Press.

KILROE P. (1994), "The grammaticalization of French à", in W. Pagliuca (ed.), Perspectives on Grammaticalization, Amsterdam/Philadelphia: John Benjamins, 49-61.

KLEIBER G. (1999), Problèmes de sémantique. La polysémie 
en question, Villeneuve d'Ascq: Presses Universitaires du Septentrion.

KLEIBER G. (2008), " Petit essai pour montrer que la polysémie n'est pas un sens interdit ", in J. Durand, B. Habert \& B. Laks (éds), Congrès Mondial de Linguistique Française - CMLF 2008, Paris : Institut de Linguistique Française, 87-101.

KOCH P. (2001), "Metonymy: Unity in diversity”, Journal of Historical Pragmatics 2 (2), 201-244.

KOCH P. (2004), "Metonymy between pragmatics, reference and diachrony", Metaphorik.de 07, 6-54. [http://www.metaphorik.de]

LIVER R. (1982), Manuel pratique de romanche (Sursilvan Vallader), Chur : Lia Rumantscha.

LURAGHI S. (2005), "Paths of Semantic Extension -From Cause to Beneficiary and Purpose", in M. Fortescue et al. (éds), Historical Linguistics 2003, Amsterdam/Philadelphia: Benjamins, 141-157.

MARCHELLO-NIZIA C. (1974), Histoire de la langue française aux XIVe et XVe siècles, Paris : Bordas.

NUNES J. J. (1969), Compêndio de gramática histórica portuguesa, Lisbonne : Livraria Clássica (7e éd.).

RAYNOUARD F. (1977 [1838]), Lexique roman, Genève: Slatkine. [réed. Paris : Silvestre]

RINKE E. \& ELSIG M. (2010), "Quantitative evidence and diachronic syntax”, Lingua 120, 2557-2568.

RONJAT J. (1980 [1930-1941]), Grammaire istorique des parlers provençaux modernes, Genève: Slatkine \& Marseille : Laffitte.

SÄVBORG T. (1941), Étude sur le rôle de la préposition 'de' dans les expressions de lieu relatives en latin vulgaire et en ancien gallo-roman, Lundequist : C. Bloms Boktryck.

SVOROU S. (1994), The grammar of space, Amsterdam/Philadelphia: John Benjamins.

TOBLER A. \& LOMMATZSCH E. (1925), Altfranzösisches Wörterbuch, Berlin/Wiesbaden : Weidmannsche Buchhandlung.

TORRES A. \& ASSUNÇÃO A. (éds) (2000), Gramática da linguagem portuguesa (1536), Fernão de Oliveira, Lisbonne : MM.

TRAUGOTT E. \& DASHER R. (2002), Regularity in Semantic Change, Cambridge: Cambridge University Press.

VANDELOISE C. (1985), « Au-delà des descriptions géométriques et logiques de l'espace: une description fonctionnelle », Lingvisticae Investigationes 9 (1), 109-129.

VANDELOISE C. (1986), L'espace en français : sémantique des prépositions spatiales, Paris : Le Seuil.

WARTBURG W. (von) (1972-1978 [1929]), Französisches etymologisches Wörterbuch: eine Darstellung des galloromanischen Sprachschatzes, Bâle : Zbinden. [Bonn : 


\section{F. Klopop]}

Principales bases de données consultées :

BFM - Base de Français Médiéval, Lyon : UMR ICAR / CNRS

- ENS de Lyon [http://bfm.ens-lyon.fr/]

CdE - Corpus del Español, M. Davies (2002-), Corpus del Español : 100 million words, 1200s-1900s [http://www.corpusdelespanol.org/]

CdP - Corpus do português, M. Davies \& M. Ferreira (2006-), Corpus do Português: 45 million words, 1300s-1900s [http://www.corpusdoportugues.org]

CoLFIS - Corpus e Lessico di Frequenza dell'Italiano Scritto Contemporaneo [http://www.ge.ilc. cnr.it/]

CORDE - Real Academia Española : Banco de datos. Corpus diacrónico del español [http://corpus.rae.es/cordenet.html]

FRANTEXT - Atilf \& CNRS [http://www.frantext.fr/]

OVI - Opera del Vocabolario Italiano textual database, ItalNet Consortium [http://artfl-project.uchicago.edu/content/ovi] 


\begin{abstract}
In this paper, I propose a reflection on semantic evolution and polysemy, with a case study of the prepositional outcomes of Latin ante in Romance languages. I show how complex the resulting paradigms are in each language, with many forms having overlapping uses as adverbs, prepositions and conjunctions. More specifically, I try to determine to which degree the appearance of new forms can be explained by the need to disambiguate. In order to do this, I study more than fifteen forms with the help of medieval and modern corpora, in four languages (French, Italian, Spanish and Portuguese). A semantic analysis in two steps, on more than four thousand prepositional occurrences in total, enables me to bring to light differences between these language systems, and to show that the mechanism at work is not so much disambiguation as a constant semantic drift from spatial to temporal and/or notional.
\end{abstract}

Keywords: prepositions, Romance languages, polysemy, semantic evolution, corpus linguistics

\title{
RÉSUMÉ
}

Dans cet article, nous proposons une réflexion sur l'évolution sémantique et la polysémie, à partir de l'étude de formes prépositionnelles issues du latin ante dans les langues romanes. Nous montrons la complexité des paradigmes résultants dans chaque langue, avec des formes nombreuses et des emplois qui se chevauchent (adverbes, prépositions, conjonctions). Nous tentons en particulier de voir dans quelle mesure l'apparition de nouvelles formes peut être liée à un besoin de désambiguïsation. Pour ce faire, nous étudions plus d'une quinzaine de formes à partir de corpus médiévaux et modernes, dans quatre langues romanes (français, italien, espagnol, portugais). Une analyse sémantique en deux temps, opérée sur plus de quatre mille occurrences prépositionnelles au total, nous permet de mettre en évidence les différences entre ces états de langue, et de montrer qu'il ne s'agit pas tant de désambiguïsation que de glissement sémantique constant du spatial au temporel et/ou au notionnel.

Mots-clés : prépositions, langues romanes, polysémie, évolution sémantique, linguistique de corpus 